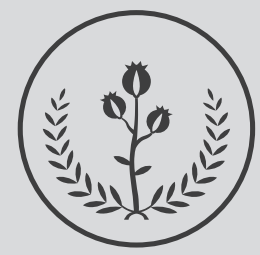

FUCS $x$

N22. 2021

\title{
Glifosato como factor de riesgo para malformaciones congénitas: revisión bibliográfica
}

Héctor Fabio Restrepo Guerrero ${ }^{a}$ Luz Helena Rincón Ruíz

\section{Glyphosate as a risk factor for congenital malformations: a literature review}

\footnotetext{
${ }^{a}$ Médico Veterinario Zootecnista. Especialista en Epidemiología. Msc Epidemiología.

Fundación Universitaria de Ciencias de la Salud. Fundación Nacional Somos- FNS, Bogotá DC, Colombia.

${ }^{b}$ Administración, Universidad del Sinú, Fundación Nacional Somos-FNS, Bogotá DC, Colombia.
}

\section{R E S U M EN}

Antecedentes: en los últimos 40 años el cambio en las prácticas agrícolas ha llevado al consecuente uso de plaguicidas con el fin de incrementar las cosechas y evitar plagas que afecten la producción, siendo un procedimiento usual a nivel mundial. En Colombia la utilización de estos plaguicidas en especial el glifosato, cuya marca comercial a nivel mundial se conoce como Roundup $^{\circledR}$, compuesto por $480 \mathrm{~g} / \mathrm{L}$ de la sal isopropilamina de $\mathrm{N}$-fosfonometilglicina, es ampliamente empleado desde hace más de 30 años sobre todo en el Valle del Cauca donde se utiliza para la maduración de la caña de azúcar y como herbicida para cultivos de café, banano, arroz, cacao y palma africana así como cítricos en otras regiones del país. Métodos: se realizó una búsqueda sistemática e integral de la literatura en las bases de datos Cochrane, Medline, Embase, Lilacs y Scielo utilizando diferentes combinaciones de exposición y términos de resultado. Resultados: la búsqueda encontró trece estudios que prueban asociaciones entre glifosato y defectos de nacimiento. Conclusiones: en cinco de los trece no se encontró asociación entre la exposición al glifosato y la presentación de malformaciones, mientras que en ocho artículos hubo algún tipo de asociación entre la presencia de malformaciones y el contacto con glifosato o sus sales. Se hace necesario realizar estudios prospectivos.

Palabras clave: glifosato, anomalías congénitas, toxicidad del desarrollo, malformaciones.

(C) 2021 Fundación Universitaria de Ciencias de la Salud -FUCS. Este es un artículo Open Access bajo la licencia CC BY-NC-ND (http:// creativecommons.org/licenses/by-nc-nd/4.0/)

INFORMACIÓN DEL ARTÍCULO

Historia del artículo:

Fecha recibido: abril 28 de 2020

Fecha aceptado: abril 8 de 2021
Autor para correspondencia. Dr. Héctor Fabio Restrepo hfrestrepo@fucsalud.edu.co
DOI

10.31260/RepertMedCir.01217372.1054 


\section{A BST R ACT}

Background: in the last 40 years, changes in agricultural practices have led to the consequent use of pesticides to increase yields and avoid pests that affect production, being a procedure commonly used worldwide. In Colombia, the use of these pesticides, especially glyphosate, whose worldwide trade name is Roundup ${ }^{\circledR}$, composed of $480 \mathrm{~g} / \mathrm{L}$ of the isopropylamine salt of N-phosphonomethylglicine, has been extensively used for more than 30 years in the Valle del Cauca region to accelerate ripening of sugar cane, and applied as a herbicide for coffee, banana, rice, cocoa and African palm crops, as well as in citrus groves, in other regions of the country. Methods: a systematic and comprehensive literature search was performed in Cochrane, Medline, Embase, Lilacs and Scielo databases using different combinations of exposure and outcome terms. Results: the search found thirteen studies testing associations between glyphosate and birth defects. Conclusions: five of the thirteen articles found no association between glyphosate exposure and the occurrence of malformations, while eight studies found some type of association between malformations and contact with glyphosate or its salts. Further prospective studies are needed.

Key words: glyphosate, congenital anomalies, developmental toxicity, malformations

(C) 2021 Fundación Universitaria de Ciencias de la Salud - FUCS. This is an open access article under the CC BY-NC-ND license (http://creativecommons.org/licenses/by-nc-nd/4.0/).

\section{INTRODUCCIÓN}

En los últimos 40 años el cambio en las prácticas agrícolas ha llevado al uso de plaguicidas con el fin de incrementar las cosechas y evitar plagas que afecten la producción, siendo una acción usual a nivel mundial. ${ }^{1-4}$ En Colombia la utilización de estos plaguicidas en especial el glifosato, cuya marca comercial a nivel mundial es conocida como Roundup $^{\circledR}$, compuesto por $480 \mathrm{~g} / \mathrm{L}$ de la sal isopropilamina de N-fosfonometilglicina ${ }^{5}$, es empleado desde hace más de 30 años sobre todo en el Valle del Cauca donde se utiliza para la maduración de la caña de azúcar y como herbicida para cultivos de café, banano, arroz, cacao, palma africana y cítricos en otras regiones del país. ${ }^{5}$ Igualmente, es el herbicida empleado en el Programa de Erradicación de Cultivos Ilícitos. ${ }^{2}$ Algunos autores consideran que se han observado efectos tóxicos en humanos en la clínica y en estudios paraclínicos ${ }^{6}$; además distintos estudios han mostrado que el glifosato es citotóxico para células tanto humanas como de otros animales. ${ }^{7-9}$ Igualmente se sugiere que el glifosato no solo se limita a la alteración del ADN en células vegetales sino que afecta también las células animales. ${ }^{4,10}$ Estudios realizados en embriones de trucha demostraron la toxicidad del glifosato produciendo muerte embrionaria en dosis de 20 partes por millón (PPM). ${ }^{11}$ Por su parte en el informe número 25 de la Fundación Ideas para la Paz revela "que la aplicación de glifosato se asoció con mortalidad en células y animales y que en humanos la exposición al producto lo fue con desenlaces como sudoración excesiva, dolor de cabeza, irritación ocular y dificultades reproductivas". ${ }^{12}$ Otros autores lo han considerado como factor de riesgo de muerte en etapas tempranas del embarazo, sin embargo este no es el tema de la presente revisión pero vale considerarlo pues está reportado en la literatura. ${ }^{13-15}$ Para 2015 el glifosato fue clasificado como posible carcinogénico por la agencia internacional para la investigación en cáncer y fue incluido en la lista de plaguicidas altamente peligrosos. ${ }^{16,17}$
En relación con el tema de las malformaciones en nuestro país, en el estudio de Zarante y col. tomaron el número de nacidos en instituciones de salud de la ciudad de Bogotá y de los 121.674 nacidos vivos en el periodo comprendido entre enero 2001 y noviembre 2010, 2.208 $(1,81 \%)$ presentaron alguna malformación evidenciada al momento del nacimiento, de los cuales $118(0,097 \%)$ eran polimalformados. ${ }^{18}$ Estas cifras dan una idea de la magnitud de nacimientos de niños con malformaciones congénitas. Richard y col. consideran que con dosis altas, inclusive en diluciones más bajas que las recomendadas en agricultura, ocurre una alteración en las células placentarias que puede llevar a problemas de tipo reproductivo. ${ }^{7}$ Por otro lado en un estudio de casos y controles donde no tuvieron en cuenta la proximidad a sitios de fumigación, Ojeda y col. observaron malformaciones en $1 \%$ de los casos, siendo las más frecuentes las relacionadas con el sistema nervioso en $29 \%$, las del aparato cardiovascular $23 \%$, vías urinarias $15 \%$, de miembros y otras en $4,5 \%$ cada uno. ${ }^{19}$

En la actualidad hay un desconocimiento sobre el mecanismo de acción del glifosato, se plantea de acuerdo con algunos autores que se produce debido a una variación en la ruta metabólica de la fosforilación oxidativa, causando un desacople de la misma con la consecuente inhibición en la formación de adenosina trifosfato, componente fundamental para el desarrollo metabólico normal. ${ }^{3,11}$ Se considera que solo $0,1 \%$ de la cantidad aplicada de plaguicidas realmente actúa sobre las plantas y el restante queda circulando en el ambiente. ${ }^{4}$

A través del tiempo ninguna investigación sobre el uso de glifosato en la aspersión ha refutado el componente tóxico del mismo. ${ }^{12}$ Sin embargo, existe gran controversia sobre los verdaderos efectos de su aplicación en la salud, sobretodo en la presentación o no de malformaciones al nacimiento, en madres que han sido expuestas a la aspersión de glifosato 
en sus etapas tempranas o tardías del embarazo. Ejemplo de esta controversia lo da el artículo publicado por Camacho y Mejía en 2015 titulado "Consecuencias de la aspersión aérea en la salud: evidencia desde el caso colombiano", publicado como capítulo del libro "Costos Económicos Y Sociales Del Conflicto En Colombia: ¿Cómo Construir Un Posconflicto Sostenible?" ${ }^{\prime 9}$ y su posterior revisión por parte de Rico y col. en 2016 titulado "Revisión de pares académicos del artículo: "Consecuencias de la aspersión aérea en la salud: Evidencia desde el caso colombiano" (Camacho y Mejía, 2015)." ${ }^{20,21}$ El primero trató el tema de las consecuencias del glifosato en la población colombiana y el segundo llevó a cabo un proceso de validación de los resultados obtenidos por el primero, lo que demuestra la gran importancia que se da al tema del glifosato y sus consecuencias en la salud. Por todos estos aspectos se hace importante en aras de intentar aclarar el papel del glifosato, llevar a cabo una revisión de la literatura en miras de conocer qué se ha investigado sobre si es o no un factor de riesgo en la presentación de malformaciones congénitas en los hijos de aquellas personas que por algún motivo han tenido contacto con el glifosato.

\section{Legislación colombiana frente al uso del glifosato}

En 1978 cuando se presentaron las primeras fumigaciones aéreas en Colombia se utilizó el herbicida llamado Paraquat. Fue retirado por presentar daños en múltiples órganos del cuerpo y gracias a la sentencia T-080 de 2017 se dejó de utilizar en Colombia. ${ }^{22}$ Sin embargo a la par de la utilización de este herbicida en 1984 el gobierno nacional autorizó el uso del glifosato, fijando límites de aplicación en búsqueda de la reducción de intoxicaciones ${ }^{23}$ y para 1994 se reglamentó oficialmente el "Programa de Erradicación de Cultivos Ilícitos mediante aspersión aérea con el herbicida glifosato (en adelante PECIG)", siendo una estrategia del Ministerio de Justicia para controlar y eliminar las plantaciones de cultivos ilícitos. $^{24}$
En 2015 y gracias a la resolución 0006 del 29 de mayo se interrumpió el uso del PECIG, debido a los posibles efectos nocivos del glifosato en la salud no solo humana sino animal y se autorizó el uso del glifosato solo para aspersiones terrestres (PECAT) por mediación de la Policía Nacional y su dirección de antinarcóticos. ${ }^{25,26}$

\section{METODOLOGÍA}

Se llevó a cabo una revisión sistemática de la literatura disponible a nivel mundial tomando los principales buscadores: The Cochrane, Medline, Embase, Lilacs y Scielo. Las ecuaciones de búsqueda que se emplearon fueron: "glyphosate" and "abnormalities congenital", "glyphosate AND developmental toxicity", "glyphosate AND birth defects", "glyphosate AND congenital anomalies", "glyphosate AND embryotoxicity", "glyphosate AND pregnancy", "glyphosate AND teratogenicity", "glyphosate AND reproductive outcomes", "glyphosate and abortions", "glyphosate AND malformations" y "glyphosate AND pregnancy outcomes". Con estas se procuró abarcar todas las posibles combinaciones para poder encontrar la mayor cantidad de artículos (tabla 1).

Los artículos incluidos cumplen los siguientes criterios de inclusión: idioma inglés o español, desarrollado en humanos, publicados desde 2000 hasta 2019, que traten sobre malformaciones congénitas, que se refieren al glifosato o sus sales y sin importar el tipo de metodología desarrollada.

Posteriormente se llevó a cabo la revisión de los resúmenes para ver el cumplimiento de criterios de inclusión. Luego se recuperaron los artículos en su totalidad para llevar a cabo la revisión de los mismos, clasificándolos de acuerdo con su pertinencia con el trabajo de investigación.

Tabla 1. Numero de artículo por términos mesh

\begin{tabular}{|l|c|c|}
\hline \multicolumn{1}{|c|}{ Glyphosate and "developmental toxicity" } & 55 & Humanos \\
Glyphosate and birth defects & 69 & 16 \\
Glyphosate and congenital anomalies & 16 & 14 \\
Glyphosate and embryotoxicity & 10 & 8 \\
Glyphosate and pregnancy & 54 & 23 \\
Glyphosate and teratogenicity & 7 & 1 \\
Glyphosate and reproductive outcomes & 16 & 9 \\
Glyphosate and abortions & 6 & 4 \\
Glyphosate and malformations & 75 & 17 \\
Glyphosate and pregnancy outcomes & 13 & 10 \\
Glyphosate" and "abnormalities congenital & 7 & 0 \\
\hline
\end{tabular}

Fuente: Ios autores. 


\section{RESULTA DOS}

Luego de la búsqueda de la literatura se obtuvieron 328 artículos, de los cuales 102 eran en humanos, el resto en animales o in vitro. Al revisar se encontró que 32 tenían más de 10 años, 29 eran repetidos y 37 contemplaban temas diferentes a los del objetivo de la presente revisión (figura 1).

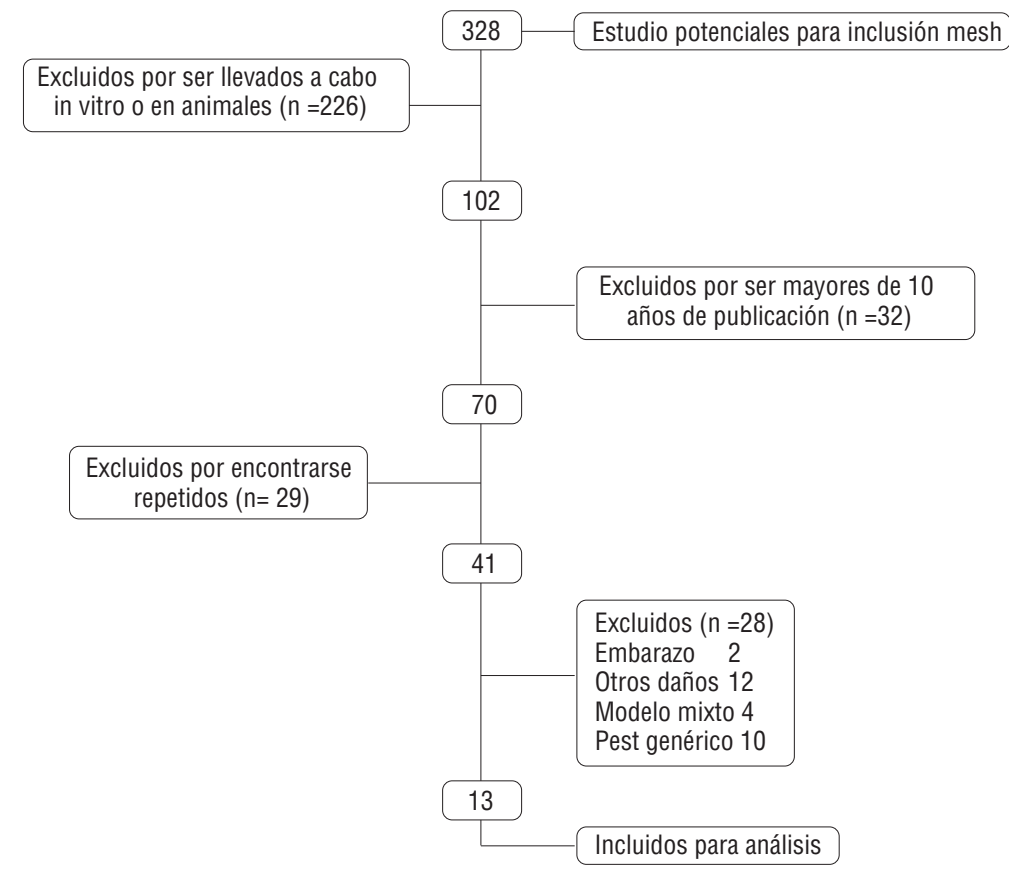

Figura 1. Diagrama de flujo del estudio. Proceso de selección de los estudios. Fuente: los autores.

\section{Análisis bibliométrico}

Después de retirar los que no cumplían con los criterios de inclusión quedaron 41 artículos, de estos se excluyeron 28 por referirse a modelos mixtos $14.3 \%$, problemas con tiempos para quedar en embarazo $7.1 \%$, otros situaciones diferentes a malformaciones congénitas $42.6 \%$ y sin especificar el tipo de pesticida $35.7 \%$. Finalmente se incluyeron 13 que se presentan en la tabla 2, en ellos el número de autores varió entre 1 y 9 viéndose representatividad de autoras femeninas en $49 \%$, el mayor número de citaciones lo tiene el artículo escrito por Garry y col. ${ }^{27}$ con 302 y los menores uno por Dutra y col. ${ }^{28}$ y otro de Franco y col. ${ }^{29}$ con 0 citaciones (fecha de consulta febrero 2020) (tabla 3).

\section{Análisis de los artículos ingresados}

Entre los trece artículos incluidos en el análisis final, el de Williams y col. no reveló efectos en la salud reproductiva o la descendencia luego de la exposición al glifosato y consideraron que los efectos no son daños de forma directa sino que fue por el resultado de los tensioactivos; tampoco hallaron literatura que soportara la implicación del glifosato como causal de malformaciones. ${ }^{30}$ McQueen y col. por su parte concluyeron que en las muestras de alimentos aunque se encuentran niveles de glifosato, estas concentraciones son bajas no siendo perjudicial para presentar malformaciones de tipo congénito. ${ }^{31}$ Así mismo De Araujo y col. luego de sus análisis de expuestos y no expuestos encontraron que "la asociación de GLY con NTD no fue significativa cuando se adoptó un modelo de pesticidas múltiples en el análisis de regresión logística $(\mathrm{OR}=1.5 ; 0.82 .9)^{\prime \prime}$, en conclusión ellos no encontraron una asociación causal entre la exposición de los padres a glifosato antes o durante el embarazo y la presentación de defectos congénitos. ${ }^{15}$

Carmichael y col. luego de llevar a cabo un estudio de casos y controles evaluando la influencia de la proximidad de zonas de aspersión (un radio de 500 metros), con madres embarazadas en estadio temprano y su repercusión en el nacimiento de niños, luego de la recolección de información no encontraron asociación entre el glifosato u otro pesticida con un riesgo mayor de producirse esta malformación $\mathrm{OR}=$ $0,88(0,48-1,64) .{ }^{32}$ Shaw y col. encontraron luego de realizar un estudio de casos y controles cuyo objetivo era determinar si las exposiciones a pesticidas en gestaciones tempranas se asociaban con el riesgo de gastrosquisis, no encontraron ninguna asociación (OR ajustado $=0,9$, ( IC 0,6-1,5). ${ }^{33}$ Yang y col. al investigar la exposición materna durante el periodo concepcional y el aumento del riesgo de presentación de labio leporino, paladar hendido o hendidura orofacial, no encontraron asociación con el glifosato. Sin embargo, hay que aclarar que no es significativo pues sus intervalos 
Tabla 2. Estudios incluidos en el análisis

\begin{tabular}{|c|c|c|c|}
\hline Titulo & Autores & Revista & Referencia \\
\hline $\begin{array}{l}\text { Birth defects, season of conception, and sex of } \\
\text { children born to pesticide applicators living in } \\
\text { the Red River Valley of Minnesota, USA.(27) }\end{array}$ & $\begin{array}{l}\text { Garry VF, Harkins ME, Erickson LL, } \\
\text { Long-Simpson LK, Holland SE, } \\
\text { Burroughs BL. }\end{array}$ & Environ Health Perspect & $\begin{array}{l}\text { Environ Health Perspect. } 2002 \text { Jun; } 110 \\
\text { Suppl 3:441-9. }\end{array}$ \\
\hline $\begin{array}{l}\text { Developmental and reproductive outcomes in } \\
\text { humans and animals after glyphosate exposure: } \\
\text { a critical analysis(30). }\end{array}$ & $\begin{array}{l}\text { Williams AL, Watson RE, De Sesso } \\
\text { JM. }\end{array}$ & J Toxicol Environ Health & $\begin{array}{l}\text { Rev. 2012;15(1):39-96. doi: } \\
\text { 10.1080/10937404.2012.632361. } \\
\text { Review. }\end{array}$ \\
\hline $\begin{array}{l}\text { Estimating maternal and prenatal exposure to } \\
\text { glyphosate in the community setting.(31) }\end{array}$ & $\begin{array}{l}\text { McQueen H, Callan AC, Hinwood } \\
\text { AL. }\end{array}$ & Int J Hyg Environ Healt & $\begin{array}{l}2012 \text { Nov;215(6):570-6. doi: } \\
\text { 10.1016/j.ijheh.2011.12.002. Epub } \\
2012 \text { Jan } 18 .\end{array}$ \\
\hline $\begin{array}{l}\text { Glyphosate and adverse pregnancy outcomes, a } \\
\text { systematic review of observational studies.(15) }\end{array}$ & $\begin{array}{l}\text { De Araujo JS, Delgado IF, } \\
\text { Paumgartten FJ. }\end{array}$ & BMC Public Health & $\begin{array}{l}\text { 2016 Jun 6;16:472. doi: } \\
\text { 10.1186/s12889-016-3153-3. Review. }\end{array}$ \\
\hline $\begin{array}{l}\text { Hypospadias and residential proximity to } \\
\text { pesticide applications.(32) }\end{array}$ & $\begin{array}{l}\text { Carmichael SL, Yang W, Roberts } \\
\text { EM, Kegley SE, Wolff C, Guo L, } \\
\text { Lammer EJ, English P, Shaw GM. }\end{array}$ & Pediatrics & $\begin{array}{l}2013 \text { Nov;132(5):e1216-26. doi: } \\
\text { 10.1542/peds.2013-1429. Epub } 2013 \\
\text { Oct 28. PMID: 24167181; PMCID: } \\
\text { PMC3813401. }\end{array}$ \\
\hline $\begin{array}{l}\text { Residential agricultural pesticide exposures and } \\
\text { risk of neural tube defects and orofacial clefts } \\
\text { among offspring in the San Joaquin Valley of } \\
\text { California. (33) }\end{array}$ & $\begin{array}{l}\text { Yang W, Carmichael SL, Roberts } \\
\text { EM, Kegley SE, Padula AM, English } \\
\text { PB, Shaw GM. }\end{array}$ & Am J Epidemiol. & $\begin{array}{l}014 \text { Mar 15;179(6):740-8. doi: } \\
\text { 10.1093/aje/kwt324. Epub } 2014 \text { Feb } 18 . \\
\text { PMID: 24553680; PMCID: } \\
\text { PMC4271111. }\end{array}$ \\
\hline $\begin{array}{l}\text { Early pregnancy agricultural pesticide exposures } \\
\text { and risk of gastroschisis among offspring in the } \\
\text { San Joaquin Valley of California.(34) }\end{array}$ & $\begin{array}{l}\text { Shaw GM, Yang W, Roberts E, } \\
\text { Kegley SE, Padula A, English PB, } \\
\text { Carmichael SL. }\end{array}$ & Clin Mol Teratol. & $\begin{array}{l}2014 \text { Sep;100(9):686-94. doi: } \\
\text { 10.1002/bdra.23263. Epub } 2014 \text { Jun } 7 .\end{array}$ \\
\hline $\begin{array}{l}\text { Congenital Malformations Associated with } \\
\text { Agrotoxics(35) }\end{array}$ & $\begin{array}{l}\text { Benitez-Leite S, Macchi ML, Acosta } \\
\text { M }\end{array}$ & Rev. chil. pediatr & $\begin{array}{l}\text { Rev. chil. pediatr. [Internet]. } 2009 \text { Ago } \\
\text { [citado } 2020 \text { Feb 07]; } 80 \text { (4): } 377-378\end{array}$ \\
\hline $\begin{array}{l}\text { Maternal residential exposure to specific } \\
\text { agricultural pesticide active ingredients and birth } \\
\text { defects in a 2003-2005 North Carolina birth } \\
\text { cohort(36) }\end{array}$ & $\begin{array}{l}\text { Rappazzo KM, Warren JL, Davalos } \\
\text { AD, Meyer RE, Sanders AP, } \\
\text { Brownstein NC, Luben TJ. }\end{array}$ & Birth Defects Res & $\begin{array}{l}\text { Birth Defects Res. } 2019 \text { Apr } \\
\text { 1;111(6):312-323. doi: } 10.1002 / \mathrm{b}- \\
\text { dr2.1448. Epub 2018 Dec } 28 .\end{array}$ \\
\hline $\begin{array}{l}\text { Teratogenic Effects of Glyphosate-Based } \\
\text { Herbicides: Divergence of Regulatory Decisions } \\
\text { from Scientific Evidence( } 37)\end{array}$ & $\begin{array}{l}\text { Fagan, John; Antoniou, Michael; } \\
\text { Habib, Mem; Howard, Cv; Jennings, } \\
\text { Rc et all }\end{array}$ & $\begin{array}{l}\text { ournal of Environmental } \\
\text { and Analytical Toxicology }\end{array}$ & S4. 10.4172/2161-0525.S4-006. \\
\hline $\begin{array}{l}\text { Identification of congenital malformations } \\
\text { associated with endocrine disrupting pesticides } \\
\text { in Brazilian grain producing states(38) }\end{array}$ & Dutra LS, Pacheco Ferreira A & $\begin{array}{l}\text { Revista Gerencia y Políticas } \\
\text { de Salud. }\end{array}$ & $\begin{array}{l}\text { 2019:18(36). } \\
\text { https://doi.org/10.11144/Javeriana.r- } \\
\text { gps18-36.imcp }\end{array}$ \\
\hline Glifosato ¿inocuo para la vida animal?(29) & $\begin{array}{l}\text { Fabián Franco, Nidia Viviana Ruiz } \\
\text { Prieto Roni Paredes }\end{array}$ & $\begin{array}{l}\text { Revista Novapolis } \\
\text { Correo Científico Médico }\end{array}$ & $\begin{array}{l}\text { № 14, Diciembre 2018, pp. 125-134. } \\
\text { Asunción: Arandurã Editorial. ISSN } \\
2077-5172 .\end{array}$ \\
\hline $\begin{array}{l}\text { Malformaciones congénitas relacionadas con los } \\
\text { agentes teratógenos(39) }\end{array}$ & $\begin{array}{l}\text { Valdés SY; Sánchez Ramírez, E; } \\
\text { Fuentes Arencibia, S. }\end{array}$ & & ccm 22( 4$): 652-666$ \\
\hline
\end{tabular}

Fuente: los autores.

Tabla 3. Características de los artículo ingresados

\begin{tabular}{|c|c|c|c|c|c|}
\hline Autor & Revista & Año publicación & Número autores & Autores mujeres & Número de citaciones* \\
\hline Garry et all & Environ Health Perspect & 2002 & 6 & 4 & 302 \\
\hline Williams et all & J Toxicol Environ Health & 2012 & 3 & 2 & 120 \\
\hline McQueen et all & Int J Hyg Environ Healt & 2012 & 3 & 2 & 23 \\
\hline De Araujo et all & BMC Public Health & 2016 & 3 & 2 & 33 \\
\hline Carmichael et all & Pediatrics & 2013 & 9 & 3 & 41 \\
\hline Yang et all & Am J Epidemiol & 2014 & 7 & 4 & 42 \\
\hline Shaw et all & Clin Mol Teratol. & 2014 & 7 & 4 & 15 \\
\hline Benitez-Leite & Rev. chil. Pediatr & 2009 & 3 & & 113 \\
\hline Rappazzo et all & Birth Defects Res & 2019 & 7 & 3 & 22 \\
\hline Fagan et all & $\begin{array}{l}\text { Journal of Environmental } \\
\text { and Analytical Toxicology }\end{array}$ & 2012 & 8 & 3 & 52 \\
\hline Dutra et all & $\begin{array}{l}\text { Revista Gerencia y } \\
\text { Políticas de Salud. }\end{array}$ & 2019 & 2 & 1 & 0 \\
\hline Franco et all & Revista Novapolis & 2019 & 2 & 1 & 0 \\
\hline Valdez et all & Correo Científico Médico & 2018 & 3 & 2 & 1 \\
\hline
\end{tabular}

Fuente: Ios autores. 
de confianza son amplios. Entre las malformaciones encontradas están anencefalia con un valor $\mathrm{OR}=0.9$ (IC 0.5-1.9), espina bífida OR =0.9, (IC $0.5-1.4)$, labio leporino con y sin paladar hendido OR $=0.9$ (IC 0.7-1.3) y paladar hendido solo OR $=0.9$ (IC 0.5-1). ${ }^{34}$ Por su parte Garry y col. luego de realizar su investigación concluyen que posterior a la identificación de 14 pesticidas, el glifosato y la fosfina mostraron una correlación con el aumento de efectos adversos. ${ }^{27}$ Benítez y col. hallaron una asociación entre la exposición a plaguicidas (glifosato) y la aparición de malformaciones congénitas en neonatos nacidos en Paraguay. ${ }^{35}$ Rapazzo y col. encontraron que los defectos de nacimiento variaban de acuerdo con las combinaciones de pesticidas a las cuales las madres estuvieron expuestas, siendo mayor en el caso del glifosato para alteraciones o defecto septal auricular. El estudio realizado en Carolina del Norte por Fagan y col ${ }^{36}$ mostró que existe evidencia que el glifosato o su nombre comercial Roundup llevan a producir efectos teratogénicos en la reproducción, como también efectos a nivel genómico que conllevan a malformaciones de tipo congénito. ${ }^{37}$ Franco y col. informaron cómo el glifosato afecta no solo el crecimiento de las malezas sino también el de la flora cercana, pero más aún afecta tanto el desarrollo intrauterino, el crecimiento neural periférico y el normal funcionamiento hormonal. ${ }^{29}$ Dutra y col. reportaron por medio de pruebas de Odds ratio una asociación estadística que confirma el aumento de número de malformaciones congénitas con la exposición a los plaguicidas entre los cuales está el glifosato. ${ }^{38}$ De acuerdo con lo encontrado por Valdes y col. en una investigación del laboratorio de embriología molecular del Conitec-UBA, aún con dosis más bajas de las que se utilizan en fumigación de soya se pueden presentar efectos a nivel de embriones alterando el desarrollo normal de los mismos, llevando a presencia de malformaciones neuronales, intestinales y cardiacas, al igual que la presencia de microcefalia y deformidades craneofaciales entre otras. ${ }^{39}$

\section{CONCLUSIONES}

Cinco de los trece artículos (Williams y col., McQueen y col., De Araujo y col., Carmichael y col. y Shawy col.) no encontraron una asociación entre la exposición al glifosato y la presentación de malformaciones, mientras que ocho artículos (Yang y col., Garry y col., Benitez y col., Rapazzo y col., Dutra y col., Franco y col., Fagan y col., Valdes y col.) hallaron algún tipo de asociación entre la presencia de malformaciones y el contacto con glifosato o sus sales. En la literatura internacional se encuentra poco sobre los efectos del glifosato en la presentación de malformaciones congénitas como se pudo comprobar en esta investigación, lo que lleva a generar más protocolos de tipo prospectivo específicos para cada uno de los pesticidas más utilizados en el mundo. Aunque existe un gran número de literatura gris que contempla las malformaciones que produce el uso de glifosato, nuestro estudio no encontró ninguno concluyente al respecto, siendo solo un estudio en el que se pudo encontrar un aumento del riesgo de presentación27, no siendo este concluyente. No es posible dar una generalización sobre los resultados encontrados en este estudio puesto que presenta una gran heterogeneidad. La revisión revela que existen cerca de 12 estudios que contemplan la presentación de malformaciones congénitas por exposición a pesticidas, pero no hacen una discriminación del glifosato sino que es genérico, lo que ocasiona la necesidad de llevar a cabo estudios más específicos para cada uno de los pesticidas que son utilizados.

\section{CONFLICTO DE INTERÉS}

Los autores declaran NO tener ningún conflicto de interese, incluidos los políticos y/o financiera asociada con patentes o propiedad, el suministro de los proveedores de materiales y/o suministros y equipos utilizados en el estudio. La financiación del estudio fue de fuentes propias.

\section{REFEREN CIAS}

1. Plenge-Tellechea F, Sierra-Fonseca JA, Castillo-Sosa YA. Riesgos a la salud humana causados por plaguicidas. Tecnociencia Chihuahua. 2007;1(3):4-6.

2. Gonzalez Plazas S. El Programa de Erradicación de Cultivos Ilícitos Mediante aspersion Aérea de Glifosato: hacia la clarificación de la política y su debate. Bogotá: Editorial Universidad del Rosario; 2006.

3. Solomon KR, Anadón A, Cerdeira AL, Marshall J, Limited M, Sanín LH. Estudio de los efectos del Programa de Erradicación de Cultivos Ilícitos mediante la aspersión aérea con el herbicida Glifosato (PECIG) y de los cultivos ilícitos en la salud humana y en el medio ambiente. Washington: División de la Organización de los Estados Americanos (OEA); 2005.

4. Aiassa D, Mañas F, Bosch B, Peralta L, Gentile N, Bevilacqua S, et al. Los plaguicidas. su relacion con la salud humana y ambiental en la provincia de Cordoba. Rev Exp Médica. 2009;27(2):39-44.

5. Varona M, Henao GL, Díaz S, Lancheros A, et al. Evaluación de los efectos del glifosato y otros plaguicidas en la salud humana en zonas objeto del programa de erradicación de cultivos ilícitos. Biomédica. 2009;29(3):456-75. doi: 10.7705/biomedica.v29i3.16

6. Martínez A, Reyes I, Reyes N. Citotoxicidad del glifosato en células mononucleares de sangre periférica humana. Biomédica. 2007;27(4):594604. doi: 10.7705/biomedica.v27i4.176

7. Cells and Aromatase. Environ Health Perspect. 2005;113(6):716-20. doi: 10.1289/ehp.7728

8. Lioi MB, Scarfi MR, Santoro A, Barbieri R, Zeni O, Di Berardino $\mathrm{D}$, et al. Genotoxicity and oxidative stress induced by pesticide exposure in bovine lymphocyte cultures in vitro. Mutat Res. 1998;403(1-2):13-20. doi: 10.1016/s0027$5107(98) 00010-4$ 
9. Arias MA, Camacho A, Ibáñez AM, Mejía D, Rodríguez C. Costos económicos y sociales del conflicto en Colombia: ¿Cómo construir un posconflicto sostenible? Colombia: Universidad de los Andes; 2014.

10. Monroy CM, Cortés AC, Sicard DM. Citotoxicidad y genotoxicidad en células humanas expuestas in vitro a glifosato. Biomédica. 2005;25(3):335-80. doi: 10.7705/biomedica.v25i3.1358

11. Ucros Garrido AM. Efectos del glifosato sobre el desarrollo embrionario de la trucha arco iris (Onchorhynchus mykiss)[Tesis]. Bogotá: Pontificia Universidad Javeriana; 2009.

12. Rico DM, Scoppetta O, Alzate JP, González Ferro A. Verdades científicas sobre glifosato y salud pública. Bogotá: Fundación Ideas para la Paz; 2016. p. 76.

13. Savitz DA, Arbuckle T, Kaczor D, Curtis KM. Male pesticide exposure and pregnancy outcome. Am J Epidemiol. 1997;146(12):1025-36. doi: 10.1093/oxfordjournals.aje.a009231

14. Arbuckle TE, Lin Z, Mery LS. An exploratory analysis of the effect of pesticide exposure on the risk of spontaneous abortion in an Ontario farm population. Environ Health Perspect. 2001;109(8):851-7. doi: 10.1289/ehp.01109851

15. De Araujo JS., Delgado IF, Paumgartten FJR. Glyphosate and adverse pregnancy outcomes, a systematic review of observational studies. BMC Public Health. 2016;16:472. doi: 10.1186/s12889016-3153-3

16. Bejarano González F, Aguilera Márquez D, Álvarez Solís J, Arámbula Meraz E, Arellano Aguilar O, Bastidas Bastidas P, et al. Los plaguicidas altamente peligrosos en México. México: Red de Acción sobre Plaguicidas y Alternativas en México, A. C. (RAPAM); 2017. p. 364.

17. Ponce, G. (2017) Sojización y perfiles de mortalidad: el caso de la Provincia de Buenos Aires entre los años 2008-2012 [Tesis de Maestría]. Argentina: Universidad Nacional de Lanús. Departamento de Salud Comunitaria; 2016.

18. Zarante AM, Gracia G, Zarante I. Evaluación de factores de riesgo asociados con malformaciones congénitas en el programa de vigilancia epidemiológica de malformaciones congénitas (ECLAMC) en Bogotá entre 2001 y 2010. Univ Méd Bogotá. 2012;53(1):11-25.

19. Ojeda LC, Benítez Leite S. Factores de riesgo prenatales y su asociación a malformaciones congénitas en un Hospital Universitario de Referencia. Pediatría (Asunción). 2018;45(1):8-15.

20. Camacho A, Mejía D. Consecuencias de la aspersion aerea en la salud: evidencia desde el caso colombiano. Bogota; 2015. p. 21.

21. Rico DM, Scoppetta O, Cuéllar D. Revision de pares academicos del articulo: "Consecuencias de la aspersión aérea en la salud: Evidencia desde el caso colombiano" (Camacho y Mejía, 2015). Bogotá: Fundación Ideas para la Paz; 2016. p. 34.

22. Corte Constitucional de Colombia. T-080-17 derecho fundamental a la consulta previa-caso en que comunidades étnicas solicitan que se detenga definitivamente la fumigación de cultivos ilícitos mediante la aspersión aérea de glifosato, sobre los territorios consagrados a la autoridad y soberanía del pueblo [Internet]. [citado 2020 Marzo 6]. Disponible en: https://www.corteconstitucional. gov.co/relatoria/2017/t-080-17.htm
23. Olasolo H. La respuesta al fenómeno del narcotráfico en Colombia mediante fumigaciones aéreas de cultivos ilícitos: análisis de legalidad a la luz del Derecho Internacional Humanitario vLex Global. Rev Costarric derecho Int [Internet]. 2015;(Citado diciembre 2015). Disponible en: https://papers.ssrn.com/sol3/ papers.cfm?abstract_id=2753911

24. Bernal Caceres K tatiana. Fumigación copn glifosato en Colombia: politica antidrogas vs. derecho a la salud y al medio ambiente. Universidad Catolica de Colombia; 2019.

25. Manrique Zuluaga V, Carmona Alert P. Glifosato ¿en consiste el debate?. Observatorio Iberoamericano de Cultivos y Drogas Ilícitas (OICDI); 2019. p. 25.

26. Consejo Nacional de Estupefacientes. Resolución 0006 de 2015. Se establecen los criterios para el pago de horas extras a los servidores públicos de la Agencia Nacional de Minería, Resolución 0006 de 2015 (2016).

27. Garry VF, Harkins ME, Erickson LL, Long-Simpson LK, Holland SE, Burroughs BL. Birth Defects, Season of Conception, and Sex of Children Born to Pesticide Applicators Living in the Red River Valley of Minnesota, USA. Environ Health Perspect. 2002;110Suppl3(Suppl 3):441-9. doi: 10.1289/ehp.02110s3441

28. Dutra LS, Pacheco Ferreira A. Identificación de malformaciones congénitas asociadas a plaguicidas disruptores endocrinos en estados brasileños productores de granos. Rev Gerenc y Políticas Salud. 2019;18(36):1-26. doi: 10.11144/Javeriana.rgps18-36.imcp

29. Franco F, Ruiz Prieto NV. Glifosato ¿ inocuo para la vida animal?. NOVAPOLIS. 2018;14:125-34.

30. Williams AL, Watson RE, Desesso JM. Developmental and reproductive outcomes in humans and animals after glyphosate exposure: A critical analysis. J Toxicol Environ Heal - Part B Crit Rev. 2012;15(1):39-96. doi: 10.1080/10937404.2012.632361

31. McQueen H, Callan AC, Hinwood AL. Estimating maternal and prenatal exposure to glyphosate in the community setting. Int J Hyg Environ Health. 2012;215(6):570-6. doi: 10.1016/j. ijheh.2011.12.002

32. Carmichael SL, Yang W, Roberts EM, Kegley SE, Wolff C, Guo $\mathrm{L}$, et al. Hypospadias and residential proximity to pesticide applications. Pediatrics. 2013;132(5):e1216-26. doi: 10.1542/ peds.2013-1429

33. Yang W, Carmichael SL, Roberts EM, Kegley SE, Padula AM, English PB, et al. Residential agricultural pesticide exposures and risk of neural tube defects and orofacial clefts among offspring in the San Joaquin Valley of California. Am J Epidemiol. 2014;179(6):740-8. doi: 10.1093/aje/kwt324

34. Shaw MG, Wei Y, Eric R, Susan EK, Amy P, Paul BE and, et al. Early Pregnancy Agricultural Pesticide Exposures and Risk of Gastroschisis among Offspring in the San Joaquin Valley of California. Birth Defects Res A Clin Mol Teratol. 100(9):686-94. doi: 10.1002/bdra.23263

35. Benítez-Leite S, Macchi ML, Acosta M. Malformaciones congénitas asociadas a agrotóxicos. Rev chil pediatr. 2007;80(4):377-78. doi: $10.4067 /$ S037041062009000400010 
36. Rappazzo KM, Warren JL, Davalos AD, Meyer RE, Sanders AP, Brownstein NC, et al. Maternal residential exposure to specific agricultural pesticide active ingredients and birth defects in a 20032005 North Carolina birth cohort. Birth Defects Res. 2019;111(6):31223. doi: $10.1002 /$ bdr2.1448

37. Robinson C. Teratogenic Effects of Glyphosate-Based Herbicides: Divergence of Regulatory Decisions from Scientific Evidence. J Environ Anal Toxicol. 2012;S4:006. doi:10.4172/2161-0525.S4-006
38. CanalS andrea C, Cavada GC, nazer julio. Factores de riesgo de ocurrencia y gravedad de malformaciones congénitas Identification of risk factors for congenital malformations. Rev Med Chile. 2014;142(11): 1431-1439. doi: 10.4067/S0034-98872014001100010

39. Valdés Silva Y, Sánchez Ramírez E, Fuentes Arencibia S. Malformaciones congénitas relacionadas con los agentes teratógenos. Correo Científico Médico. 2018;22(4):652-66. 